\title{
USO da TÉCniCa de Análise de FlutuaÇões COM RemoÇão de TendênCias PARA O ESTUdO de ESTRELAS VARIÁVEIS.
}

\author{
Bianca Ramielly Bomfim de Jesus ${ }^{1}$; Marildo Geraldête Pereira ${ }^{2}$. \\ 1.Bolsista FAPESB, Graduanda em Física, Universidade Estadual de Feira de Santana, \\ email:biancaramielly@hotmail.com \\ 2.Orientador, Departamento de Física, Universidade Estadual de Feira de Santana, \\ email:marildogpegmail.com \\ PALAVRAS-CHAVE: Variabilidade Estelar, Fotometria, DFA.
}

\section{INTRODUÇÃO}

Estrelas variáveis são objetos de grande interesse na astrofísica, pois as mesmas nos permitem extrair várias informações sobre os processos físicos e as condições em que estes acontecem. A variabilidade estelar é uma das mais populares e dinâmicas áreas da astronomia, por conta de ser uma propriedade comum a maioria das estrelas. Ela nos provê com certos parâmetros, dentre eles: escalas temporais e amplitudes. Estes parâmetros, os quais são disponíveis apenas para estrelas com esta característica, podem ser utilizados para extrair propriedades físicas, como: massa, luminosidade, rotação, entre outros. $\mathrm{O}$ estudo da variabilidade nos permite a observação direta das mudanças que ocorrem nas estrelas, indo das mais rápidas e violentas, relacionadas com o nascimento e morte de estrelas, até as mudanças mais lentas, relacionadas com a evolução estelar normal [Percy 2007].

$\mathrm{Na}$ astronomia observacional, os fenômenos de variabilidade podem ser observados por meio da fotometria da radiação eletromagnética proveniente da estrela, esta é então transformada em uma série temporal, denominada de curva de luz, que descreve a variação do brilho da fonte em função do tempo. Tais curvas de luz, podem ser analisadas por diversos métodos, o que dependerá dos objetos a serem estudados.

Este trabalho está focado primeiramente no estudo de duas classes de estrelas: a Sub anãs do tipo B ( $\mathrm{SdB})$ e O (SdO) e as Anãs Brancas (WDs). As SdBs e SdOs representam uma das fases evolutivas das estrelas de alta massa. Sendo encontradas entre a sequencia principal e as anãs brancas no diagrama Hertzprung-Russel (HR) (Figura 1) [Heber 2009].

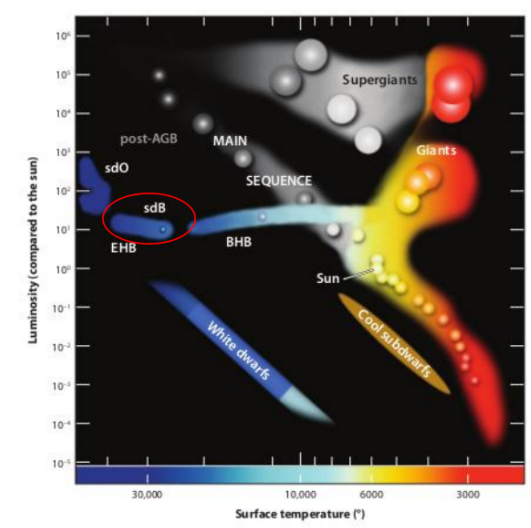

Figura 1: Diagrama HR, com destaque nas estrelas $\mathrm{SdB}$, no estremo horizontal azul. [Heber 2009] 
As estrelas anãs brancas são o estágio final da maioria das estrelas. Estima-se que mais de 97\% das estrelas, incluindo o nosso sol se tornem WDs. Assim sendo, estas estrelas apresentam importantes informações a respeito da evolução de estrelas individuais [Althaus et al. 2010].

Lançado em 2009, o satélite Kepler, com sua estratégia observacional apresentou grandes oportunidades de observações de estrelas variáveis devido ao seu modo de operação e monitoramento. Com seu campo de observação fixado na direção da constelação do Cisne, sua estratégia de observação permitiu que estrelas variáveis pudessem ser observadas por longos períodos (meses-anos) com elevada resolução temporal ( $1 \mathrm{~min}$ ), permitindo assim que uma vasta casta de objetos variáveis fossem pela primeira vez observados e descobertos. As características dessas observações são adequadas para estudos de variabilidade fotométrica, de forma que uma abordagem usando a Análise de Flutuações com Remoção de Tendências (do inglês Detrended Fluctuation Analysis - DFA) se mostra adequada para este tipo de observações, como já demonstrado em trabalhos anteriores [Zebende et al. 2005, Zebende, Fernandez e Pereira 2016]. Os dados públicos do Kepler podem ser encontrados no Nasa Exoplanet Archive ${ }^{1}$, e podem ser encontrados no modo de longa cadência (obtém uma integração a cada 30 min), ou como usado neste trabalho, curta cadência (obtém uma integração a cada $58.85 \mathrm{~s}$ ).

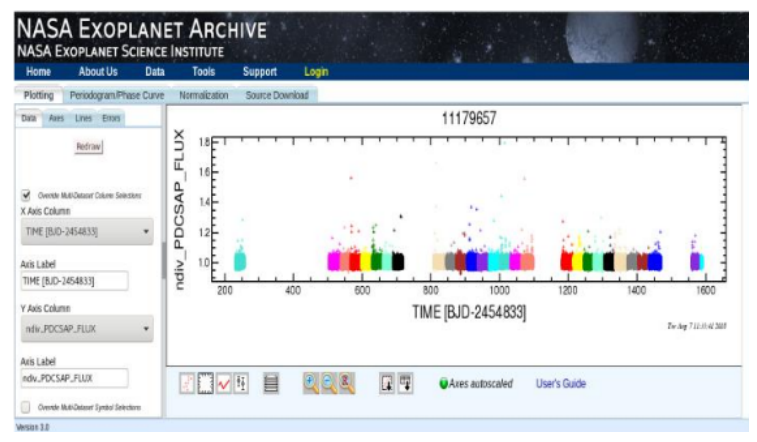

Figura 2: Exemplo de uma curva de luz normalizada por divisão, presente no banco de dados do kepler.

\section{Metodologia}

Para a análise das curvas de luz foi utilizado o método de Análise de Flutuações Depuradas de Tendências (do inglês Detrended Fluctuation Analisys - DFA). Este método foi desenvolvido por [Peng et al. 1994], e consiste em se determinar a correlação da memória de escala (ou correlação de longo alcance) de um determinado fenômeno que ocorre em uma determinada escala, no caso deste trabalho uma escala de tempo.

Ao aplicar o método $\mathrm{DFA}^{2}$ às curvas de luz, o programa gera um gráfico, conforme observado na Figura 3. Após isto, para cada escala temporal relacionada com um coeficiente de correlação $\alpha$ são determinados os limites temporais de variabilidade para aquele dado coeficiente.

\footnotetext{
${ }^{1}$ https://exoplanetarchive.ipac.caltech.edu/

${ }^{2} \mathrm{O}$ código DFA pode ser encontrado em: https://www.physionet.org/physiotools/dfa/
} 


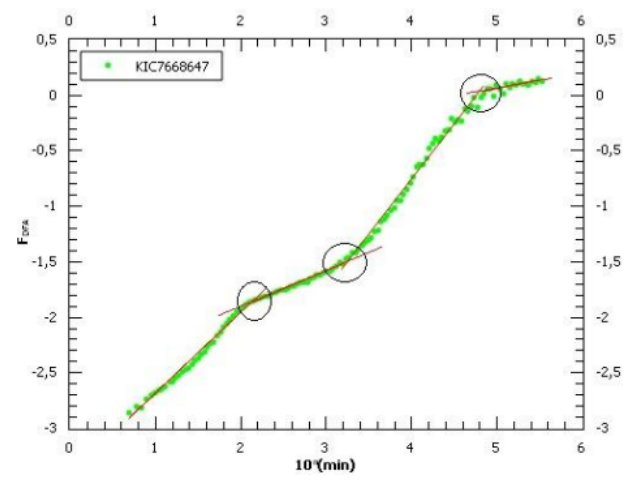

Figura 3: Curva de Luz após o uso do DFA, mostrando a regressão linear das suas partes lineares, sendo destacadas as suas intersecções.

No gráfico contido na Figura 3, pode-se encontrar o $\alpha$ DFA a partir da regressão linear das partes lineares entre as intersecções. A intersecção das linhas contínuas em $F_{D F A}(n)$ representa o limite da transição de um tipo de variabilidade para outra, e o valor do expoente $\alpha_{D F A}$ comprova este fato [Zebende, Fernandez e Pereira 2016].

\section{Resultados}

Para uma primeira análise foram simuladas (em linguagem de programação R) duas séries temporais, as quais apresentam um comportamento de Ruído Branco e Ruído Browniano, sendo então feita uma análise DFA para ambas as séries. Fazendo esta análise podemos relacionar o coeficiente de correlação $\alpha$ com os resultados a serem obtidos com a análise das séries temporais das fontes a serem analisadas.

Os coeficientes de correlação encontrados para as duas séries temporais, $\alpha=0,49$ para o ruído branco e 1,5 para o ruído browniano estão de acordo com os valores encontrados em literatura [Peng et al. 1995], com $\alpha \sim 0,5$ para um série que apresenta um comportamento característico de ruído branco e $\alpha \sim 1,5$ para uma série com comportamento de ruído browniano.

Por meio da investigação sobre fontes estelares do tipo sdB e WD observadas pelo Satélite Kepler, encontradas na literatura [Zong 2016, McNamara, Jackiewicz e McKeever 2012], de acordo com os critérios de terem uma cobertura temporal grande e observadas no modo de curta cadência, mostradas nas tabelas 1 e 2 .

Tabela 1: Fontes WD

\begin{tabular}{|l|c|}
\hline KIC1718290 & KIC10536147 \\
\hline KIC2697388 & KIC10799291 \\
\hline KIC3544662 & KIC11302565 \\
\hline KIC6866662 & KIC11953267 \\
\hline KIC8619526 & KIC7540755 \\
\hline KIC9020774 & \\
\hline
\end{tabular}


Tabela 2: Fontes sdB pulsantes

\begin{tabular}{|l|c|}
\hline KIC0267388 & KIC10139564 \\
\hline KIC02991276 & KIC10553698 \\
\hline KIC02991403 & KIC10670103 \\
\hline KIC03527751 & KIC11179657 \\
\hline KIC05807616 & KIC11558725 \\
\hline KIC07664467 & KIC02438324(B4) \\
\hline KIC07668647 & KIC08302197 \\
\hline KIC10001893 & \\
\hline
\end{tabular}

\section{Conclusão}

Neste trabalho foi feita uma simulação de variabilidades associadas com uma série aleatória, caracterizada por ruído branco, e uma associada com variabilidade browniana, encontrando- se os valores para os coeficientes de correlação de longa escala de acordo com a literatura. Também foi feito um levantamento de 26 fontes, sendo 15, estrelas sub anãs quentes e 11 estrelas anãs brancas, à serem analisadas futuramente.

\section{Referências}

ALTHAUS, L. G. et al. Evolutionary and pulsational properties of white dwarf stars. The Astronomy and Astrophysics Review, Springer, v. 18, n. 4, p. 471-566, 2010.

HEBER, U. Hot subdwarf stars. Annual review of Astronomy and Astrophysics, Annual Reviews, v. 47, p. 211-251, 2009.

MCNAMARA, B. J.; JACKIEWICZ, J.; MCKEEVER, J. The classification of kepler b-star variables. The Astronomical Journal, IOP Publishing, v. 143, n. 4, p. 101, 2012.

PENG, C.-K. et al. Mosaic organization of dna nucleotides. Physical review e, APS, v. 49, n. 2, p. $1685,1994$.

PENG, C.-K. et al. Quantification of scaling exponents and crossover phenomena in nonstationary heartbeat time series. Chaos: An Interdisciplinary Journal of Nonlinear Science, AIP, v. 5, n. 1, p. 82-87, 1995.

PERCY, J. R. Understanding variable stars. [S.1.]: Cambridge University Press, 2007.

ZEBENDE, G. et al. Universal persistence in astrophysical sources. Physica A: Statistical Mechanics and its Applications, Elsevier, v. 349, n. 3-4, p. 452-458, 2005.

ZEBENDE, G. F.; FERNANDEZ, B. F.; PEREIRA, M. G. Analysis of the variability in the sdb star kic 10670103: Dfa approach. Monthly Notices of the Royal Astronomical Society, Oxford University Press, v. 464, n. 3, p. 2638-2642, 2016.

ZONG, W. Amplitude and frequency modulations of oscillation modes in hot B subdwarf and white dwarf stars from Kepler photometry. Tese (Doutorado) - Université de Toulouse, Université Toulouse III-Paul Sabatier, 2016. 